\title{
Peri-threshold Trigeminal Stimulation with Capsaicin Increases Taste Sensitivity in Humans
}

\author{
Pengfei Han ${ }^{1,2,3}$ - Lea Müller ${ }^{1} \cdot$ Thomas Hummel $^{1}$
}

Received: 8 December 2020 / Accepted: 1 June 2021 / Published online: 1 July 2021

(c) The Author(s) 2021

\begin{abstract}
Introduction Taste perception is affected by trigeminal stimuli, i.e., capsaicin. This has been studied at suprathreshold concentrations. However, little is known about taste perception at threshold level in the presence of low concentration of capsaicin. The aim of the study was to explore whether taste sensitivity for sweet, sour, salt, bitter, and umami is modulated by the presence of capsaicin in the peri-threshold range.

Methods Fifty-seven adults (age range 19-85 years; 32 women) with functional gustation participated in the study. Based on their perception of phenylthiocarbamide (PTC), the group was stratified into non-tasters $(n=20)$ and tasters $(n=37)$. Threshold for sweet (sucrose), sour (citric acid), salty (sodium chloride), bitter (quinine-hydrochloride), and umami (sodiumglutamate) tastes was estimated using a single-staircase paradigm (3-alternative forced choice; volume per trial $0.1 \mathrm{ml}$ ) with or without $0.9-\mu \mathrm{M}$ capsaicin added. This capsaicin concentration had been determined in pilot studies to be in the range of oral perception thresholds.

Results The addition of capsaicin produced lower taste thresholds for sweet, sour, salty, and bitter but not for umami. In contrast, neither PTC taster status nor sex affected these results.

Conclusion The current results indicate that a low concentration of capsaicin increases gustatory sensitivity.

Implications The current findings provide evidence supporting different effects of capsaicin on taste perception at threshold level. It has implications for boosting taste sensitivity or flavor enjoyment with low concentration of capsaicin.
\end{abstract}

Keywords Taste $\cdot$ Irritation $\cdot$ Trigeminal $\cdot$ Mouth $\cdot$ Psychophysics $\cdot$ Thresholds

\section{Introduction}

Growing evidence has shown that the perception of sensory cue from one modality is influenced by the simultaneous activation of other sensory modalities (Verhagen and Engelen 2006). The oral trigeminal-mediated sensation (including

Pengfei Han

p.han@foxmail.com

Lea Müller

lea.mueller2@tu-dresden.de

Thomas Hummel

thummel@msx.tu-dresden.de

1 Interdisciplinary Center On Smell and Taste, Department of Otorhinolaryngology, TU Dresden, Dresden, Germany

2 Faculty of Psychology, Southwest University, Chongqing, China

3 Key Laboratory of Cognition and Personality, Ministry of Education, Chongqing, China mechanical, thermal, chemical, and pain sensations) can affect taste perception (Braud and Boucher 2020; Mistretta and Bradley 2021). Trigeminal stimuli arising from food intake may impact on flavor perception. Certain foods are good examples of the trigeminal-taste interaction, such as carbonated sweetened beverages (Hewson et al. 2009) or spicy chili dishes. The most studied trigeminal stimulant is capsaicin, which is the main trigeminally active compound in chili pepper. Capsaicin produces irritative sensations such as tickling, stinging, burning, heat, or pain.

Previous studies have described suppressive effect of capsaicin (either in a capsaicin-taste mixture or prior mouth rinse) on the intensity of sweet, bitter, salty, and umami tastes in humans (Green and Hayes 2003; Green and Schullery 2003; Lawless and Stevens 1984; Narukawa et al. 2011; Prescott and Stevenson 1995; Simons et al. 2002), with few showing no effect of capsaicin on salty taste intensity (Lawless and Stevens 1984; Prescott et al. 1993). Conversely, studies have also demonstrated the effect of taste on the 
capsaicin-induced burning sensation. Prescott et al. (1993) showed the burning sensation produced by capsaicin solution $(6.5 \mu \mathrm{M}, 13.1 \mu \mathrm{M}$, or $26 \mu \mathrm{M})$ was uninfluenced by sucrose $(0.1-0.4 \mathrm{M})$, but the burning sensation of $13.1-\mu \mathrm{M}$ capsaicin was increased by $\mathrm{NaCl}(0.075-0.3 \mathrm{M})$, and that the effect of $\mathrm{NaCl}$ on burning sensation varied according to the capsaicin concentrations. However, another study showed that the presence of sweet taste decreases capsaicin irritation by approximately $50 \%$, and the effect can be partially reversed by adding a sweet taste inhibitor (lactisole) (Smutzer et al. 2018). This results indicate that the effect of sucrose on capsaicin perception is mediated by the sweet taste receptor (Smutzer et al. 2018). In addition, several studies found suprathreshold level of capsaicin can elicit bitter (Green and Schullery 2003; Green and Hayes 2004; Just et al. 2007) or salty taste (Lawless 1984) sensations, although only found in part of the study participants and fade away shortly after capsaicin application. In fact, the co-localization of capsaicin receptors (vanilloid receptor subtype 1) with sweet or bitter taste receptors has been found in rat (Moon et al. 2010) and human (Jahng et al. 2010) taste papillae.

While extensive research had been done regarding the capsaicin-taste interaction at suprathreshold level, less has been investigated when both are presented at threshold levels. Taste threshold is defined as the lowest concentration of a compound that can be discriminated or recognized from the solvent. Both reflecting gustatory functions, the threshold (sensitivity) and suprathreshold intensity were poorly correlated for basic tastes, indicating each individual measure characterizes a separate component of the taste perception (Bartoshuk 1978; Keast and Roper 2007). Correlations were found between the sensitivity and preferences for certain types of taste in humans (Chamoun et al. 2019), suggesting the possibility of change taste (sensory)-based food preferences through taste sensitivity modulation. A few previous studies have demonstrated the threshold for capsaicin based on aqueous solutions, with a threshold range between 0.29 and $1.14 \mu \mathrm{M}$ (in a 10-ml volume) (Lawless et al. 2000; Sizer and Harris 1985). Moreover, the coexistence of capsaicin receptors with sweet and bitter taste receptors in papillae (Jahng et al. 2010; Moon et al. 2010) also provides a molecular basis for potential interactions between stimuli from the two modalities at threshold levels (Mistretta and Bradley 2021).

Therefore, the primary aim of this study was to investigate the impact of capsaicin on taste sensitivity. Specifically, recognition thresholds of sweet (sucrose), salty (sodium chloride; $\mathrm{NaCl}$ ), bitter (quinine-hydrochloride), sour (citric acid), and umami (monosodium glutamate, MSG) were assessed with and without addition of very low concentrations of capsaicin (peri-threshold level). Moreover, since the perceived capsaicin-induced irritation intensity differs as a function of the participants' inherited sensitivity to bitter compounds (Prescott and Swain-Campbell 2000), the current study was also designed to explore the influence of phenylthiocarbamide (PTC) taster status on the postulated effects of capsaicin on taste sensitivity.

\section{Materials and Method}

\section{Participants}

Fifty-seven non-smoking healthy subjects participated (age mean $=33.0$ years, $\mathrm{SD}=17.8$; range from 19 to 85 years; 25 men, 32 women; $\mathrm{BMI}$ mean $=22.2, \mathrm{SD}=2.7$, range from 16.7 to 29.5). Participants' gustatory function was screened via taste sprays that consist of suprathreshold concentrations (diluted in 100-ml distilled water) of "sweet" (10-g D-saccharose), "sour" (5-g citric acid), "salty" (7.5-g NaCl), and "bitter" (0.025-g quinine-hydrochloride) solutions (Hummel et al. 2013). All participants were able to identify each of the four tastes correctly. Participants were asked to place a $3.80 \mathrm{~cm} \times 1.43 \mathrm{~cm}$ strip of filter paper impregnated with $0.007 \mathrm{mg}$ of phenylthiocarbamide (PTC; Sensonics, Inc., $\mathrm{NJ}, \mathrm{USA}$ ) in the mouth for a minimum of $5 \mathrm{~s}$. Individuals who rated the bitterness intensity with score $1=$ "barely detectable" were considered "non-tasters" (NT; $n=20$, age mean $=45.8$ years, $\mathrm{SD}=22.4 ; 7$ women), whereas those who detect a bitter sensation (with ratings of 2 weak, 3 moderate, 4 strong, or 5 extremely strong) were classified as "tasters" ( $\mathrm{T} ; n=37$, age mean $=26.1$ years; $\mathrm{SD}=9.4$; 25 women) (Bartoshuk et al. 1994). Participants (Table 1) reported whether they eat hot spices with a yes-or-no question. The study was conducted in accordance with the Declaration of Helsinki and its design was approved by the Ethics Committee at the TU Dresden. The power calculation using G*Power tool software (Faul et al. 2007) showed that a sample size in the present study of $n=57$ would be adequate to detect significant differences (Wilcoxon signed-rank test, $a=0.05$, power $=0.8$ ) given a medium effect size of $d=0.4$.

Table 1 Participants information of this study

\begin{tabular}{|c|c|c|c|}
\hline Category & Subcategory & $\begin{array}{l}\text { No. of } \\
\text { participant }\end{array}$ & Percentage \\
\hline \multirow[t]{2}{*}{ Gender } & Females & 32 & $56.1 \%$ \\
\hline & Males & 25 & $43.9 \%$ \\
\hline \multirow[t]{2}{*}{ PTC taste status } & Taster & 37 & $64.9 \%$ \\
\hline & Non-taster & 20 & $35.1 \%$ \\
\hline \multirow[t]{2}{*}{ Spicy food consumption ${ }^{2}$} & Yes & 20 & $35.1 \%$ \\
\hline & No & 37 & $64.9 \%$ \\
\hline
\end{tabular}




\section{Capsaicin Concentration}

A pilot test was carried out with 12 subjects who were not involved in later experiments to determine the capsaicin concentration which would not yield apparent trigeminal responses and to be used for taste sensitivity tests. Because the capsaicin is insoluble in water, the samples were prepared in 95\% ethanol solutions. We dissolve the capsaicin (analytical standard of $\geq 99.0 \%$ by HPLC; Sigma-Aldrich, Steinheim, Germany; order number 12084) with ethanol first, and then we diluted the capsaicin-ethanol solution further into the water to reach the final concentrations, with the final ethanol concentration at a level imperceptible to participants (below $0.5 \%$ ). A concentration of $0.9-\mu \mathrm{M}$ capsaicin was determined to be in the range of peri-threshold level. The concentration was also comparable to that shown in previous research (Bovelet et al. 2015; Narukawa et al. 2011), which the capsaicin concentrations were $0.5 \mu \mathrm{M}, 1 \mu \mathrm{M}$, and $0.9 \mu \mathrm{M}$, respectively.

\section{Taste Sensitivity Test}

Gustatory recognition threshold was assessed in solutions with or without added capsaicin for five basic tastes: sweet (sucrose), sour (citric acid), salty $(\mathrm{NaCl})$, bitter (quininehydrochloric acid), and umami (MSG). For each taste, six dilutions were prepared based on the work of Gudziol and Hummel (2007), and were identified using equivalence numbers 1 to 6 with highest concentration given to number " 6 " and lowest concentration given to number " 1 " (Table 2). Two series of dilutions were prepared for each taste. These differed only in the composition of the solvent. One was made with the solvent (distilled water) containing minute amounts of ethanol $(0.00275 \%)$; these amounts of ethanol did not produce a noticeable taste when tried at volumes of $0.1 \mathrm{ml}$. The other was made with the solvent (distilled water with the $0.00275 \%$ alcohol $)$ added with capsaicin $(0.9 \mu \mathrm{M})$. All prepared samples were kept at room temperature and protected from light. All dilution series were tested per subject, i.e. the five series without capsaicin and the five series

Table 2 Molar concentrations for taste solutions under each dilution step

\begin{tabular}{llllll}
\hline $\begin{array}{l}\text { Dilution } \\
\text { steps }\end{array}$ & $\begin{array}{l}\text { Sucrose } \\
(\mathrm{mM})\end{array}$ & $\begin{array}{l}\text { Citric acid } \\
(\mathrm{mM})\end{array}$ & $\begin{array}{l}\mathrm{NaCl} \\
(\mathrm{mM})\end{array}$ & $\begin{array}{l}\text { Quinine } \\
(\mathrm{mM})\end{array}$ & $\begin{array}{l}\text { MSG } \\
(\mathrm{mM})\end{array}$ \\
\hline 6 & 21.9 & 15.6 & 41.1 & 0.133 & 17.7 \\
5 & 11.0 & 7.8 & 20.5 & 0.067 & 8.9 \\
4 & 5.5 & 3.9 & 10.3 & 0.033 & 4.4 \\
3 & 2.7 & 2.0 & 5.1 & 0.017 & 2.2 \\
2 & 1.4 & 1.0 & 2.6 & 0.008 & 1.1 \\
1 & 0.7 & 0.5 & 1.3 & 0.004 & 0.6 \\
\hline
\end{tabular}

with capsaicin in the solvent. During experiments, half of the participants received the samples without capsaicin first, and the other half received the samples with capsaicin first. The test order for different taste qualities was the same for all participants.

Participants were asked to refrain from food, drink (except water), cigarette, or chewing gums from at least 30 min prior to the test. Taste recognition threshold test was performed following a single-staircase three-alternative forced choice procedure with four reversals, in which the subjects were provided with three samples (two controls and one containing the target stimuli) per trial, in ascending order from the lowest to the highest concentrations. In brief, a pipette triplet of drops (100 $\mu \mathrm{l}$ each) comprising two drops of the solvent (ethanol-water solution or ethanol-water-capsaicin solution) and one sample drop additionally containing one of the five tastes (sweet, salty, bitter, sour, or umami) were administered sequentially onto the middle of the anterior third of the extended tongue (Gudziol and Hummel 2007). For each trial, participants were asked to choose the sample that most likely contained one of the tastes and name the taste quality (sweet, sour, bitter, salty, or umami). The taste samples were administered in increasing concentrations, starting with the highest dilution and sorted according to taste. If a wrong sample was selected, the concentration was increased in the subsequent trial until two consecutive correct answers were given, the value of which was noted as the first turning point. Then, the concentration was lowered again until subjects did not identify the taste anymore. This concentration was noted as the next turning point. Afterwards, the concentration was increased again until the taste was recognized two times in a row (third turning point) which triggered a lowering of the taste concentrations until the taste was no longer identified (forth turning point). The average of the four turning points (the corresponding taste concentrations) was used as a threshold estimate. The interval between two trials was approximately $20 \mathrm{~s}$, during which participants rinsed their mouth with tap water. During the test, if participants could not recognize the taste quality at the highest concentration (dilution step 6), they received a score of 7 (Gudziol and Hummel 2007). The total trial numbers and the whole duration of the test varied between participants, but in general, the whole test took approximately $40 \mathrm{~min}$.

\section{Ancillary Tests}

Two sequential ancillary tests were performed in a small sample $(N=10$, mean age $=26.3$ years; $\mathrm{SD}=9.4$; six females and four males) from the main experimental cohort on separate days. First, participants' recognition threshold for five tastes with no capsaicin was assessed. The first ancillary test was aimed to investigate whether irritation of the trigeminal nerve with capsaicin influences taste sensitivity 
if the irritation occurred before the actual taste sample was administered. Participants kept $2 \mathrm{ml}$ of a capsaicin solution $(0.9 \mu \mathrm{M})$ in the mouth for $4 \mathrm{~min}$ and then spat it out. After a pause of $2 \mathrm{~min}$, the same taste sensitivity test without capsaicin addition was performed again. The second ancillary test was to find out whether the capsaicin-induced irritation outside the oral cavity influences taste sensitivity. The Capsamol@ ointment (WÖRWAG PHARMA, Böblingen, Germany) with the active ingredient cayenne pepper thick extract, corresponding to capsaicinoids calculated as capsai$\operatorname{cin}(50 \mathrm{mg} / 100 \mathrm{~g}$ ointment), was used for this test. According to the package leaflet, the ointment is used for the external treatment of muscle pain. A very low dose was applied, and the test subjects were only allowed to take part in the test if their skin was intact and free of open areas. A cotton swab was dabbed with the ointment. The subject was asked to use the cotton swab to spread some ointment on the skin of the left and right cheek. A few seconds after application, the subjects stated that they felt sharp and burning, but well tolerable sensations on the skin (intensity rating of 6 or 7 on a 10-point scale). This was followed by the determination of the threshold for the sweet (sucrose) and salty (sodium chloride) tastes without added capsaicin. A selection of two tastes was considered sufficient to receive some orientation of the potential distracting influence of external capsaicin treatment.

\section{Statistical Analyses}

Data were analyzed using IBM SPSS Statistics version 21 (Chicago, IL). The effect of capsaicin on taste sensitivity was analyzed using repeated measures ANOVA. The influences of PTC taste status on taste sensitivity with and without added capsaicin were analyzed using mixed design ANOVA, with one between-subject factor (PTC taster and non-taster) and two within-subject factors (taste quality, capsaicin addition), including sex and age as co-variables of no interest. Post-hoc comparisons between independent variables were performed with Bonferroni corrections. All analyses were analyzed using SPSS (v. 21) and the significance threshold was set to $\alpha 1 / 40.05$. Effect sizes of the ANOVA terms are reported as $\eta^{2}$.

\section{Results}

\section{Effect of Capsaicin on Taste Sensitivity}

There was a significant effect of capsaicin addition $\left(F_{(1,55)}=25.1, p<0.001\right.$, partial $\left.\eta^{2}=0.31\right)$ on taste sensitivity (Fig. 1). Pairwise comparisons revealed that the addition of capsaicin significantly decreased the threshold score for sweet $(p=0.01)$, salty $(p=0.002)$, bitter $(p=0.01)$, and sour $(p=0.001)$ tastes, but not for umami $(p=0.15)$. We observed no significant interaction between taste quality and capsaicin addition on sensitivity score $\left(F_{(4,220)}=0.7, p=0.59\right)$.

\section{Effect of Oral and Cheek Capsaicin Pretreatment on Taste Sensitivity}

The ancillary tests showed that there was a weak but significant effect of oral capsaicin pretreatment on taste sensitivity (Fig. 2) $\left(F_{(1,9)}=5.13, p=0.049\right)$; however, none of the taste qualities survived the significant threshold after correction for multiple comparisons (for all $p>0.05$ ). There is no significant interaction between taste quality and oral capsaicin pretreatment on taste sensitivity $\left(F_{(4,36)}=0.26, p=0.91\right)$. In addition, compared to taste sensitivity without capsaicin, pretreatment with Capsamol ${ }^{\circledR}$ ointment on the cheek had no significant effect on taste sensitivity $\left(F_{(1,9)}=0.13, p=0.73\right)$.

\section{Factors Influencing the Effect of Capsaicin on Taste Sensitivity}

There was no significant interaction between PTC taster status and capsaicin addition on taste sensitivity $\left(F_{(1,52)}=0.34\right.$, $p=0.56)$. There was neither significant interaction between gender capsaicin on taste sensitivity.
Fig. 1 Effect of 0.9- $\mu \mathrm{M}$ capsaicin (capsaicin +) on taste recognition threshold. In each box and whisker plot, the central line denotes the median and the plus $(+)$ denotes the mean. The upper edge of the box represents the $75 \%$ quartile, the lower edge represents the $25 \%$ quartile, and the ends of the whiskers represent the maximum and minimum data points

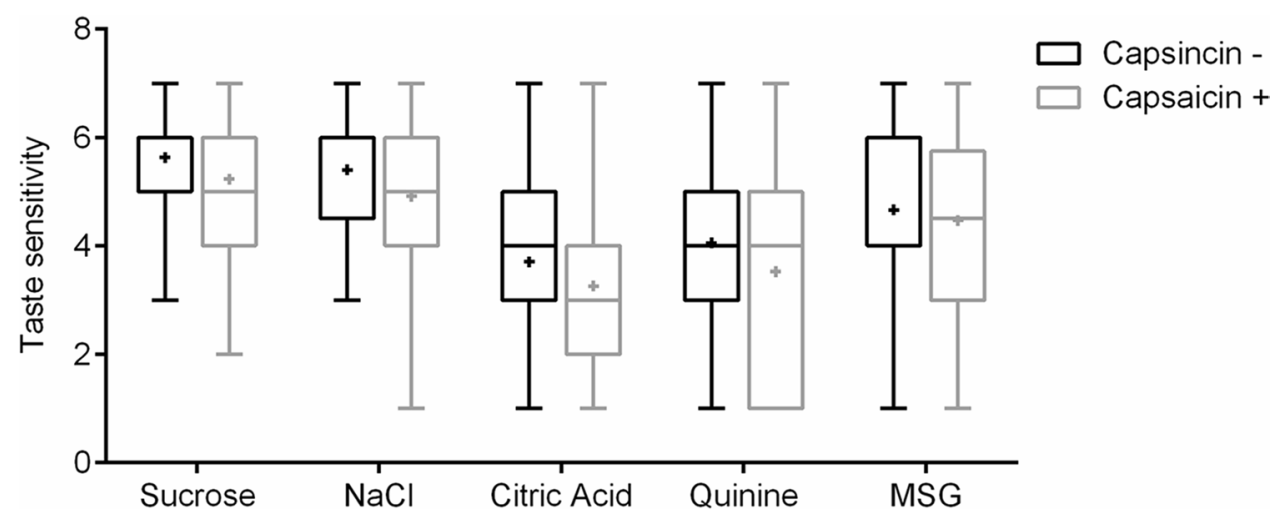




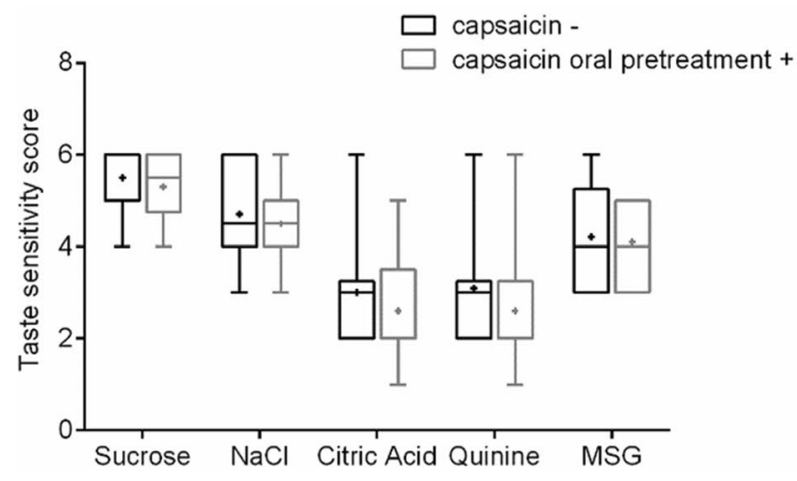

Fig. 2 Effect of oral (left panel) and cheek (right panel) pretreatment with capsaicin on taste recognition threshold in a subsample of participants $(N=10)$. In each box and whisker plot, the central line denotes the median and the plus $(+)$ denotes the mean. The upper

\section{Discussion}

Results from the current study showed that a low concentration of capsaicin increased taste sensitivity by lowering the recognition thresholds. Capsaicin may affect transmitter release, detection at a receptor level, transduction, and central-nervous integration. The capsaicin receptor (vanilloid receptor subtype 1) are co-localized with basic taste (sweet and bitter) receptors in human taste papillae (Jahng et al. 2010). Capsaicin itself is associated with bitter or sweet sensations (Green and Schullery 2003; Just et al. 2007). Besides, taste perception had been suggested to have a somatosensory component. For example, tactile stimulation of the taste papilla elicited chemical taste sensations (Cardello 1981), and strong cross-desensitization of the burning and stinging of $\mathrm{NaCl}$ and citric acid was mediated by activation of the capsaicin-sensitive fibers (Gilmore and Green 1993). Thus, capsaicin may affect taste transduction or taste receptor cell excitability and hence affect taste perception (Simons et al. 2003). Besides, oral pretreatment with capsaicin had a similar effect on decreasing taste threshold as compared to the capsaicin-taste mix, indicating a potential impact of capsaicin on taste perception, which lasts at least for several minutes. In contrary to multiple studies showing suppressive effect of capsaicin on taste intensity perception, one previous study had found that a low concentration of capsaicin $(0.5 \mu \mathrm{M}$ or $1 \mu \mathrm{M})$ increased the taste intensity of $\mathrm{NaCl}$ solution (Narukawa et al. 2011). It is likely that the concentration of capsaicin is important for its effect on capsaicin-taste binary mixture perception, which has been well-documented in taste-taste interactive effect (Wilkie and Phillips 2014). Previous study showed that capsaicin solution, when applied at high concentrations, were perceived as bitter by half of the study participants, whose perception of irritation was not different. This suggested independent transduction mechanisms for taste perception (bitter or

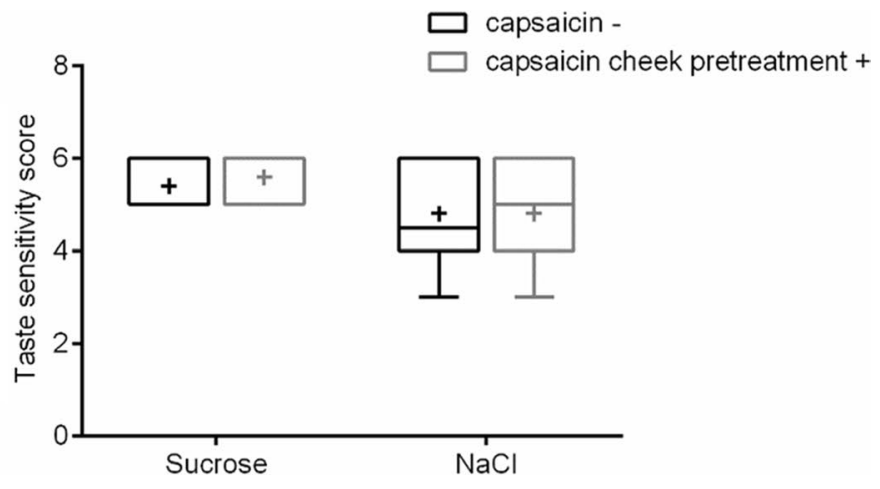

edge of the box represents the $75 \%$ quartile, the lower edge represents the $25 \%$ quartile, and the ends of the whiskers represent the maximum and minimum data points

sweet) and chemesthetic sensations (irritation) of capsaicin (Green and Hayes 2004). However, the current results may also be possibly related to the inter-papillary capsaicin-taste interaction caused by competition for receptor sites. Taken together, the trigeminal-taste cross-modal interplay could rely on multilevel interactions.

Central top-down modulation may also contribute to the effect. Brain imaging studies reported integration of capsaicin and taste perception in the anterior ventral insula (Rudenga et al. 2010). However, only limited portions of neurons in the primate's insula/operculum taste cortex respond to capsaicin stimulation (Verhagen et al. 2004). Thus, capsaicin elicited activation of the trigeminal system may gate or modulate gustatory processing at some stage in the taste pathway such as the solitary nucleus or thalamus. Presumably, the presence of trigeminal and taste stimuli increases the perceptual complexity which may produce an advantage in the processing of the gustatory information, similar to findings in bimodal odor perception (Pellegrino et al. 2017). One possible explanation may be that oral exposure to low concentrations of capsaicin produces an attentional capture of the taste sensation while high concentrations of capsaicin direct the attention away from taste to irritation that leads to a reduction of perceived intensity. However, considering the present ancillary experiments with capsaicin stimulation on the cheek, such attentional effects do not seem to be very strong. Taken together, the effect of taste sensitivity enhancement by low concentration of capsaicin could conceivably involve both peripheral and central neural interactions (Rhyu et al. 2021).

The null finding regarding the effect of PTC taster status may be due to the scaling method used for separating taster from non-tasters. A scale that is often used for determining these classifications is an adjective-labeled scale with ratio properties, such as the labeled magnitude scale (LMS) or the general labeled magnitude scale (gLMS), which were 
recommended for comparison across groups (Bartoshuk 2000; Bartoshuk et al. 2004). Categorical scales, such as the one used in the current study, may have different properties, i.e., one person's rating of 4 cannot be assumed to be twice as intense as another person's rating of 2. Having said that, an advantage of the presently used scale was that it is easily comprehended by the participants. Future studies are needed to explore differences between the two types of scales in depth.

The $0.9-\mu \mathrm{M}$ capsaicin used in the current study was determined as close to the threshold level, which means the sensation of irritation was not detectable or only barely detectable by the participants. This capsaicin concentration was comparable to the previously reported range for capsaicin threshold (0.29 to $1.14 \mu \mathrm{M}$ in a 10-ml volume) (Sizer and Harris 1985), or the group mean capsaicin threshold of $1 \mu \mathrm{M}(0.31 \mathrm{mg} / \mathrm{l}$ in a 10-ml volume) (Lawless et al. 2000). Although this concentration is higher compared to the capsaicin threshold level (e.g. a threshold between 0.16 and $0.49 \mu \mathrm{M}$ ) reported in other studies (Okamoto et al. 2018; Orellana-Escobedo et al. 2012), it is distinct from (far below) the concentration that could induce perception of irritation, for example, a concentration of around $0.36 \mu \mathrm{M}(0.11 \mathrm{ppm})$ was barely detectable, and $0.9 \mu \mathrm{M}$ of capsaicin $(0.275 \mathrm{ppm})$ was barely detectable to weak sensation (Nolden and Hayes 2017). The variability between thresholds obtained in different studies may also be explained by different modes of stimulation and especially by differences in the volume of the administered stimuli. Also, because sensitivity of capsaicin varies between individuals and may be affected by multiple factors, a direct comparison of capsaicin threshold may be inappropriate.

The increased taste sensitivity by capsaicin may have nutritional implications. For example, generic taste loss (e.g. reduced taste sensitivity) is common among elderly people (Mojet et al. 2001). In addition, the decrement in food enjoyment among patients with chemosensory dysfunctions seems to be extremely high (from 60 to $90 \%$ of the patients) (Croy et al. 2014; Mattes et al. 1990). There is an trigeminal-taste interaction in palatability processing (Berridge and Fentress 1985); therefore, the addition of low concentrations of capsaicin in food product may constitute a potential approach to boost taste sensitivity, food palatability, and the enjoyment of foods (Ludy et al. 2015).

Several limitations to the current study need to be acknowledged. First, some participants failed to recognize the taste solution at the highest concentration, which limit the sensitivity of the gustatory test; future researches with broader range of concentrations are necessary. Second, although a pilot study suggested the concentration used was in the range of thresholds, future studies may include the assessment of individual capsaicin thresholds. This might be helpful to further understand the role of conscious capsaicin perception on taste sensitivity. Additionally, the taste solutions with added capsaicin may have induced irritation among some participants whose capsaicin threshold was below $0.9 \mu \mathrm{M}$, which might be related to a negative emotional status. The emotions should be assessed and controlled in future research. Finally, the participants' age range was broad (19-85 years) which may have effect on the results; hence, the findings related to these analyses need to be validated for different age groups in future studies with large sample size.

In conclusion, the current study showed a peri-threshold concentration of capsaicin increases gustatory sensitivity when present in taste solutions. Given that taste perception at the threshold or suprathreshold levels reflect different aspects of the gustatory function (Webb et al. 2015), these findings suggested the complexity of oral perception of binary taste-trigeminal mixtures.

Funding Open Access funding enabled and organized by Projekt DEAL.

\section{Declarations}

Conflict of Interest All authors declare no competing interests.

Ethical Approval The study was carried out in accordance with the Declaration of Helsinki and was approved by the Ethics Committee of TU Dresden.

Informed Consent Written informed consent was obtained from all participants prior to the experiment.

Open Access This article is licensed under a Creative Commons Attribution 4.0 International License, which permits use, sharing, adaptation, distribution and reproduction in any medium or format, as long as you give appropriate credit to the original author(s) and the source, provide a link to the Creative Commons licence, and indicate if changes were made. The images or other third party material in this article are included in the article's Creative Commons licence, unless indicated otherwise in a credit line to the material. If material is not included in the article's Creative Commons licence and your intended use is not permitted by statutory regulation or exceeds the permitted use, you will need to obtain permission directly from the copyright holder. To view a copy of this licence, visit http://creativecommons.org/licenses/by/4.0/.

\section{References}

Bartoshuk LM (1978) The psychophysics of taste. Am J Clin Nutr 31:1068-1077

Bartoshuk LM (2000) Comparing sensory experiences across individuals: recent psychophysical advances illuminate genetic variation in taste perception. Chem Senses 25:447-460

Bartoshuk LM, Duffy VB, Miller IJ (1994) PTC/PROP tasting: anatomy, psychophysics, and sex effects. Physiol Behav 56:1165-1171

Bartoshuk LM, Duffy VB, Green BG, Hoffman HJ, Ko CW, Lucchina LA, Marks LE, Snyder DJ, Weiffenbach JM (2004) Valid acrossgroup comparisons with labeled scales: the gLMS versus magnitude matching. Physiol Behav 82:109-114 
Berridge KC, Fentress JC (1985) Trigeminal-taste interaction in palatability processing. Science 228:747-750

Bovelet P, Iannilli E, Zhigang Z, Hummel T (2015) fMRI investigation of the effects of capsaicin as taste enhancer. In Association for Chemoreception Sciences (AChemS), 37th Annual Meeting, vol. 40, ed.^eds. Oxford Univ Press, Bonita Springs, pp. 535-668

Braud A, Boucher Y (2020) Intra-oral trigeminal-mediated sensations influencing taste perception: A systematic review. J Oral Rehabil 47:258-269

Cardello AV (1981) Comparison of taste qualities elicited by tactile, electrical, and chemical stimulation of single human taste papillae. Percept Psychophys 29:163-169

Chamoun E, Liu AAS, Duizer LM, Darlington G, Duncan AM, Haines J, Ma DWL (2019) Taste sensitivity and taste preference measures are correlated in healthy young adults. Chem Senses 44:129-134

Croy I, Nordin S, Hummel T (2014) Olfactory disorders and quality of life-an updated review. Chem Senses 39:185-194

Faul F, Erdfelder E, Lang A, Buchner A (2007) G*POWER3: a flexible statistical power analysis program for the social, behavioral, and biomedical sciences. Behav Res Methods 39:175-191

Gilmore MM, Green BG (1993) Sensory irritation and taste produced by $\mathrm{NaCl}$ and citric acid: effects of capsaicin desensitization. Chem Senses 18:257-272

Green B, Hayes J (2003) Capsaicin as a probe of the relationship between bitter taste and chemesthesis. Physiol Behav 79:811-821

Green BG, Hayes JE (2004) Individual differences in perception of bitterness from capsaicin, piperine and zingerone. Chem Senses 29:53-60

Green BG, Schullery MT (2003) Stimulation of bitterness by capsaicin and menthol: differences between lingual areas innervated by the glossopharyngeal and chorda tympani nerves. Chem Senses 28:45-55

Gudziol H, Hummel T (2007) Normative values for the assessment of gustatory function using liquid tastants. Acta Otolaryngol 127:658-661

Hewson L, Hollowood T, Chandra S, Hort J (2009) Gustatory, olfactory and trigeminal interactions in a model carbonated beverage. Chemosens Percept 2:94-107

Hummel T, Hummel C, Welge-Luessen A (2013) Assessment of olfaction and gustation. In: Welge-Luessen A, Hummel T (eds) Management of smell and taste disorders: a practical guide for clinicians. Thieme, Stuttgart, pp. 58-75

Jahng JW, Moon YW, Lee J-H (2010) Immunohistochemical detection of capsaicin receptors in taste cells of human circumvallate papillae. Asian J Oral Maxillofac Surg 22:193-197

Just T, Pau HW, Steiner S, Hummel T (2007) Assessment of oral trigeminal sensitivity in humans. Eur Arch Otorhinolaryngol 264:545-551

Keast RS, Roper J (2007) A complex relationship among chemical concentration, detection threshold, and suprathreshold intensity of bitter compounds. Chem Senses 32:245-253

Lawless H (1984) Oral chemical irritation: psychophysical properties. Chem Senses 9:143-155

Lawless H, Stevens DA (1984) Effect of oral chemical irritation on taste. Physio Behav 32:995-998

Lawless HT, Hartono C, Hernandez S (2000) Thresholds and suprathreshold intensity functions for capsaicin in oil and aqueous based carriers. J Sens Stud 15:437-447

Ludy MJ, Tucker RM, Tan SY (2015) Chemosensory properties of pungent spices: their role in altering nutrient intake. Chemosens Percept 8:131-137

Mattes RD, Cowart BJ, Schiavo MA, Arnold C, Garrison B, Kare MR, Lowry LD (1990) Dietary evaluation of patients with smell and/ or taste disorders. Am J Clin Nutr 51:233-240

Mistretta CM, Bradley RM (2021) The fungiform papilla is a complex, multimodal, oral sensory organ. Curr Opin Physiol 20:165-173
Mojet J, Christ-Hazelhof E, Heidema J (2001) Taste perception with age: generic or specific losses in threshold sensitivity to the five basic tastes? Chem Senses 26:845-860

Moon YW, Lee JH, Yoo SB, Jahng JW (2010) Capsaicin receptors are colocalized with sweet/bitter receptors in the taste sensing cells of circumvallate papillae. Genes Nutr 5:251-255

Narukawa M, SasaKi S, Watanabe T (2011) Effect of Capsaicin on Salt Taste Sensitivity in Humans. Food Sci Technol Res 17:167-170

Nolden AA, Hayes JE (2017) Perceptual and affective responses to sampled capsaicin differ by reported intake. Food Qual Prefer 55:26-34

Okamoto N, Okumura M, Tadokoro O, Sogawa N, Tomida M, Kondo E (2018) Effect of single-nucleotide polymorphisms in TRPV1 on burning pain and capsaicin sensitivity in Japanese adults. Mol Pain 14:1744806918804439

Orellana-Escobedo L, Ornelas-Paz JJ, Olivas GI, Guerrero-Beltran JA, Jimenez-Castro J, Sepulveda DR (2012) Determination of absolute threshold and just noticeable difference in the sensory perception of pungency. J Food Sci 77:S135-S139

Pellegrino R, Drechsler E, Hummel C, Warr J, Hummel T (2017) Bimodal odor processing with a trigeminal component at suband suprathreshold levels. Neuroscience 363:43-49

Prescott J, Stevenson RJ (1995) Effects of oral chemical irritation on tastes and flavors in frequent and infrequent users of chili. Physiol Behav 58:1117-1127

Prescott J, Swain-Campbell N (2000) Responses to repeated oral irritation by capsaicin, cinnamaldehyde and ethanol in PROP tasters and non-tasters. Chem Senses 25:239-246

Prescott J, Allen S, Stephens L (1993) Interactions between oral chemical irritation, taste and temperature. Chem Senses 18:389-401

Rhyu MR, Kim Y, Lyall V (2021) Interactions between chemesthesis and taste: role of TRPA1 and TRPV1. Int J Mol Sci 22:3360

Rudenga K, Green B, Nachtigal D, Small DM (2010) Evidence for an integrated oral sensory module in the human anterior ventral insula. Chem Senses 35:693-703

Simons CT, O'Mahony M, Carstens E (2002) Taste suppression following lingual capsaicin pre-treatment in humans. Chem Senses 27:353-365

Simons CT, Boucher Y, Carstens E (2003) Suppression of central taste transmission by oral capsaicin. J Neurosci 23:978-985

Sizer F, Harris N (1985) The influence of common food additives and temperature on threshold perception of capsaicin. Chem Senses 10:279-286

Smutzer G, Jacob JC, Tran JT, Shah DI, Gambhir S, Devassy RK, Tran EB, Hoang BT, McCune JF (2018) Detection and modulation of capsaicin perception in the human oral cavity. Physiol Behav 194:120-131

Verhagen JV, Engelen L (2006) The neurocognitive bases of human multimodal food perception: sensory integration. Neurosci Biobehav Rev 30:613-650

Verhagen JV, Kadohisa M, Rolls ET (2004) Primate insular/opercular taste cortex: neuronal representations of the viscosity, fat texture, grittiness, temperature, and taste of foods. J Neurophysiol 92:1685-1699

Webb J, Bolhuis DP, Cicerale S, Hayes JE, Keast R (2015) The relationships between common measurements of taste function. Chemosens Percept 8:11-18

Wilkie LM, Phillips ED (2014) Heterogeneous binary interactions of taste primaries: perceptual outcomes, physiology, and future directions. Neurosci Biobehav Rev 47:70-86

Publisher's Note Springer Nature remains neutral with regard to jurisdictional claims in published maps and institutional affiliations. 